\title{
Mortalidad en pacientes hospitalizados en el servicio de medicina interna de un hospital universitario
}

\author{
Mortality in patients hospitalized in the \\ internal medicine service of a \\ university hospital
}

\author{
David Cortés-SiERra, Nini Johanna Forero-Durán, \\ Édgar Miguel Sarmiento-Reyes, Carmelo José Espinosa-Almanza \\ - Bogotá, D.C. (Colombia)
}

\section{Resumen}

Justificación: la mortalidad intrahospitalaria hace parte de los indicadores de calidad en atención en salud, su análisis permite el desarrollo de proyectos para el mejoramiento de los servicios.

Material y métodos: se realizó un estudio de corte transversal retrospectivo, evaluando los egresos hospitalarios de medicina interna del año 2015 en el Hospital Universitario Clínica San Rafael (HUCSR). Se estableció la tasa de mortalidad y sus causas. También se realizó un análisis descriptivo de las principales características sociodemográficas y clínicas de la población, la significancia estadística se estableció en $p=0.05$.

Resultados: se presentaron 152 muertes de 1817 egresos hospitalarios para una tasa de mortalidad de $8.3 \%$. El 54.7\% fueron hombres; la mediana de edad de 71 años, el rango fue de 18-106 años. Las comorbilidades más frecuentes fueron en $67 \%$ hipertensión arterial, 39\% cáncer, 32\% EPOC. No se presentaron diferencias estadísticamente significativas entre hombres y mujeres. Las principales causas de muerte fueron en $43 \%$ enfermedades infecciosas, seguida de neoplasia sólida en $27 \%$ y finalmente de origen cardiovascular en $17 \%$. La frecuencia de infección nosocomial en la población fue de $21 \%$.

Conclusiones: la mortalidad en los pacientes adultos hospitalizados por medicina interna en el HUCSR es de $8.3 \%$ y está dentro del margen reportado por la literatura. En estos pacientes es alta la frecuencia de comorbilidades como la hipertensión arterial 67\%, cáncer (39\%) y la falla cardiaca (30\%). (Acta Med Colomb 2019; 44: 11-16).

Palabras clave: mortalidad / adulto / calidad de la atención de salud (DeCS).

\footnotetext{
Abstract

Justification: in-hospital mortality is part of the quality indicators in health care. Its analysis allows the development of projects for the improvement of services.

Materials and Methods: a retrospective cross-sectional study was carried out, evaluating the internal medicine hospital discharges of the year 2015 in the Hospital Universitario Clinica San Rafael (HUCSR). The mortality rate and its causes were established. A descriptive analysis of the main socio-demographic and clinical characteristics of the population was also carried out. The statistical significance was established at $\mathrm{p}=0.05$.

Results: of 1817 hospital discharges, there were 152 deaths, for a mortality rate of $8.3 \% .54 .7 \%$ were men; median age was 71 years, the range was $18-106$ years. The most frequent comorbidities were $67 \%$ arterial hypertension, $39 \%$ cancer, $32 \%$ COPD. There were no statistically significant differences between men and women. The main causes of death were infectious diseases in $43 \%$, followed by solid neoplasia in $27 \%$ and of cardiovascular origin in $17 \%$. The frequency of nosocomial infection in the population was $21 \%$.
}

Dr. David Cortés-Sierra: Medicina Interna Fundación Universitaria Juan N Corpas; Dra. Nini Johanna Forero-Durán: Medicina Interna Fundación Universitaria Juan N Corpas; Dr. Édgar Miguel Sarmiento-Reyes: Medicina Interna y Epidemiología, Hospital Universitario Clínica San Rafael; Dr. Carmelo José EspinosaAlmanza: Medicina Interna y Epidemiología Clínica, Universidad Nacional de Colombia. Bogotá, D.C. (Colombia).

Correspondencia: Dra. Nini Johanna ForeroDurán. Bogotá, D.C. (Colombia).

E-mail: ninijofor@hotmail.com dcorts11@gmail.com

Recibido: 21/VI/2017 Aceptado: 30/I/2019 
Conclusions: mortality in adult patients hospitalized by internal medicine in the HUCSR is $8.3 \%$ and is within the range reported by the literature. In these patients, the frequency of comorbidities such as high blood pressure 67\%, cancer (39\%) and heart failure (30\%) is high. (Acta Med Colomb 2019; 44: 11-16).

Keywords: mortality, adult, quality of health care (DeCS).

\section{Introducción}

La mortalidad es uno de los principales indicadores de calidad en la atención y gestión hospitalaria; pues se considera es una medida de la efectividad de la intervención sanitaria $(1,2)$. Su análisis sistemático data de la década de 1970, en el siguiente decenio se consolida como un indicador de interés estatal y global en muchos países $(1,3)$. Sobre la mortalidad no solo es importante su frecuencia, sino también el estudio de los factores relacionados, lo que permite la elaboración de políticas en salud en torno a mejorar de forma continua la expectativa de vida de las poblaciones (4). La literatura generalmente abarca el estudio de la mortalidad desde la perspectiva de una enfermedad, sin embargo, el análisis global de su causalidad es también importante, uno de los más destacados es el reporte anual de la OMS sobre la mortalidad mundial, en él se resalta como primera causa de muerte en países sin conflicto armado las enfermedades cardiovasculares $(48 \%)$, seguidas por el cáncer $(21 \%)$, las enfermedades crónicas del pulmón $(11 \%)$ y la diabetes $(3.67 \%)(5)$.

En medicina interna las series revisadas muestran una mortalidad que varía entre 6 y $22 \%$, dicha variabilidad tan alta habla de lo distintos que pueden ser los servicios a razón de su complejidad y población de influencia $(1,2,6-12)$. En la mayoría de servicios, sin embargo, las principales causas de muerte en general son: la cardiopatía isquémica, la EPOC, la neumonía como causa infecciosa y finalmente el cáncer, estas causas varían también en orden por grupos de edad y complejidad hospitalaria $(1,3,6,8-10)$. Así mismo no solamente es importante conocer las causas directas de mortalidad de estos pacientes, sino tipificar las comorbilidades que los acompañan, analizando factores de riesgo condicionantes que contribuyen al desenlace en la población. Entidades como hipertensión arterial 50-65\%, diabetes mellitus $10-20 \%$ (8), falla cardiaca $20 \%$ (13), EPOC $17-24 \%$ (1) y cáncer $13 \%(6,13)$, siguen siendo las más reportadas. Dada la importancia del conocimiento del indicador mortalidad en los diferentes sistemas sanitarios, nuestra investigación pretende establecer la tasa de mortalidad general del servicio de medicina interna del Hospital
Universitario Clínica San Rafael y describir las principales características clínicas presentes en los pacientes.

\section{Material y métodos}

Se realizó un estudio de corte transversal sobre la mortalidad de los pacientes egresados por al departamento de medicina interna del Hospital Universitario Clínica San Rafael en el año 2015. La recolección de los datos se realizó de forma retrospectiva usando el departamento de archivo del hospital, se solicitaron todos los certificados de defunción que habían tenido lugar en el año 2015 y luego con el uso del software informático Heon, se realizó la revisión de cada una de las historias para la extracción de la información. Para quedar incluidos en el estudio los pacientes debían tener historia clínica completa, haber estado hospitalizados a cargo de medicina interna, tener una edad mayor de 16 años y una estancia hospitalaria de al menos 24 horas. Se excluyeron a las mujeres embarazadas, los pacientes remitidos para procedimientos quirúrgicos o por complicaciones de estos y los pacientes provenientes de la unidad de cuidado intensivo que fallecieran antes de 24 horas del traslado al piso. La base de datos fue desarrollada y construida en el paquete informático Excel 2014, su procesamiento y análisis se llevó a cabo en el software estadístico STATA 14.0. Las variables cualitativas se resumieron en porcentajes, las variables cuantitativas en medias, medianas y desviaciones estándar (14). El grupo de pacientes fue adicionalmente dividido entre hombres y mujeres, las comparaciones para variables cuantitativas se realizaron usando una prueba $t$ - student o un Wilcoxon rank sum test dependiendo de la normalidad en la distribución. Las comparaciones para variables cualitativas se realizaron con un test de chi2 o una prueba exacta de Fisher según los valores esperados en cada caso. El nivel de significancia se determinó siempre a dos colas con un valor p menor de 0.05 (15).

\section{Resultados}

La cantidad de egresos hospitalarios del departamento de medicina interna durante el año 2015 fue de 1817 pacientes, de los cuales 152 fallecieron, arrojando una tasa de muerte

Convención de palabras: OMS (Organización Mundial de la Salud), EPOC (Enfermedad pulmonar obstructiva crónica), IAM (Infarto agudo de miocardio), ACV (Accidente cerebrovascular), ICC (Insuficiencia Cardiaca Congestiva), ERC (Enfermedad Renal Crónica), TRR (Terapia de Remplazo Renal), DE: (Desviación estándar), NYHA: (New York Heart Association), VIH: (Virus de Inmunodeficiencia Humana) HAART: (Highly Antiretroviral Therapy), HTA: (Hipertensión arterial); CKD-EPI: (Chronic Kidney Disease - Epidemiology Collaboration), IVU: (Infección de Vías Urinarias). 
de $8.3 \%$. Entre los fallecimientos 83 eran hombres $(54.7 \%)$ y 69 mujeres (45.3\%), la edad mínima fue 18, la máxima 106, con mediana de edad de 71 años, el $77 \%$ de los pacientes tenían más de 60 años a su ingreso. Respecto a la procedencia de la población, 81 pacientes venían de localidades al sur de la ciudad (53.3\%) las cuales en su mayoría están en los estratos 1, 2 y 3 . Ocho de fuera de la ciudad (25\%), la estancia hospitalaria media de los pacientes fallecidos fue de 22 días con una DE de $\pm 18,1$. Sobre las comorbilidades: $67 \%$ de los pacientes tenía hipertensión arterial sistémica, seguida del antecedente de cáncer en $39 \%$, EPOC en $32 \%$, falla cardiaca en $30 \%$, diabetes mellitus en $28 \%$ y ERC estadio 5 en $9 \%$. Se destaca también la necesidad de uso de oxígeno domiciliario en $22 \%$ y la presencia de infección por virus VIH en cinco casos (3.9\%), de los cuales todos fueron hombres. Entre las comorbilidades más frecuentes de acuerdo con el género, estas fueron: en las mujeres: diabetes mellitus $33 \%$ versus $24 \%$ en hombres y la neoplasia $47 \%$ versus $28 \%$ en hombres. En los hombres fue más frecuente la presencia de falla cardiaca $36 \%$ versus $24 \%$ en mujeres y la cirrosis $10 \%$ versus $4 \%$ en mujeres. Estas diferencias sin embargo no fueron estadísticamente significativas $(p>$ $0.05)$. La carga de comorbilidades fue evaluada también por escala de Charlson, $64 \%$ de pacientes tenían puntajes de tres puntos, la clase funcional fue evaluada por NYHA con clase III o más en $25 \%$ de las mujeres respecto de III o más en 33\% de los hombres. Se identificó que 69 pacientes (45.4\%) habían tenido una hospitalización en los últimos tres meses, en su mayoría (79\%) en salas generales.

Entre los medicamentos, $42.8 \%$ de los fallecidos venia tomando por lo menos un medicamento perteneciente al grupo farmacoterapéutico de alto riesgo (dentro de los cuales se mencionan los anticoagulantes orales, hipoglucemiantes, antiagregantes y citostáticos, entre otros), los más frecuentes fueron anticoagulantes con 21 pacientes $(32.3 \%)$ seguido por esteroides con 16 pacientes $(24.6 \%)$. No se presentaron diferencias significativas entre hombres y mujeres en los niveles de hemoglobina, albúmina sérica, creatinina sérica y glucosa sérica al ingreso hospitalario. Para un mayor detalle de las características de la población y su comparación observe la Tabla 1 .

La principal causa de muerte en la población fue la de origen infeccioso $(43 \%)$ seguida de la neoplasia sólida (27\%) y en tercer lugar la cardiovascular (17\%). Se presentaron con mucha menor frecuencia como causas de muerte, ACV en $7 \%$ y EPOC en $3 \%$, los valores detallados por grupos y el

Tabla 1. Características de la población.

\begin{tabular}{|c|c|c|c|c|}
\hline Variable & Hombres & Mujeres & Total & Pvalor \\
\hline Edad $( \pm D E)$ & $70.9( \pm 19.2)$ & $71.7( \pm 16.9)$ & $71.3(18.1)$ & 0.78 \\
\hline \multicolumn{5}{|l|}{ Comorbilidades } \\
\hline Hipertensión arterial (\%) & $55(66 \%)$ & $47(68 \%)$ & $102(67 \%)$ & 0.83 \\
\hline Diabetes tipo $2(\%)$ & $20(24 \%)$ & $23(33 \%)$ & $43(28 \%)$ & 0.52 \\
\hline Terapia de reemplazo renal (\%) & $9(11 \%)$ & $5(7 \%)$ & $14(9 \%)$ & 0.85 \\
\hline Neoplasia (\%) & $23(33 \%)$ & $29(48 \%)$ & $60(39 \%)$ & 0.13 \\
\hline Insuficiencia cardiaca $(\%)$ & $30(36 \%)$ & $17(25 \%)$ & $47(31 \%)$ & 0.39 \\
\hline EPOC $(\%)$ & $32(39 \%)$ & $18(26 \%)$ & $50(33 \%)$ & 0.39 \\
\hline Cirrosis (\%) & $6(7 \%)$ & $3(4 \%)$ & $9(6 \%)$ & 0.86 \\
\hline Infección HIV (\%) & $5(6 \%)$ & $0(0 \%)$ & $5(3 \%)$ & ---- \\
\hline \multicolumn{5}{|l|}{ Paraclínicos al ingreso } \\
\hline Hemoglobina $( \pm D E)$ & $11.9 \mathrm{~g} / \mathrm{dL}( \pm 3.0)$ & $11.8 \mathrm{~g} / \mathrm{dL}( \pm 2.8)$ & $11.9 \mathrm{~g} / \mathrm{dL}( \pm 3.0)$ & 0.86 \\
\hline Plaquetas $( \pm D E)$ & $236000( \pm 113.647)$ & $287000( \pm 175.772)$ & $259234( \pm 146.891)$ & 0.03 \\
\hline Glucosa $( \pm D E)$ & $133 \mathrm{mg} / \mathrm{dL}( \pm 77.1)$ & $150 \mathrm{mg} / \mathrm{dL}( \pm 86.4)$ & $141 \mathrm{mg} / \mathrm{dL}( \pm 81.6)$ & 0.27 \\
\hline Creatinina $( \pm D E)$ & $2.04 \mathrm{mg} / \mathrm{dL}( \pm 2.1)$ & $1.62 \mathrm{mg} / \mathrm{dL}( \pm 1.7)$ & $1.86 \mathrm{mg} / \mathrm{dL}( \pm 1.9)$ & 0.18 \\
\hline Albúmina $( \pm D E)$ & $2.59 \mathrm{~g} / \mathrm{dL}( \pm 0.7)$ & $2.78 \mathrm{~g} / \mathrm{dL}( \pm 0.6)$ & $2.68( \pm 0.7)$ & 0.26 \\
\hline \multicolumn{5}{|l|}{ Desenlaces en la estancia } \\
\hline Días de estancia $( \pm D E)$ & $21.7( \pm 15.6)$ & $22.3( \pm 20.9)$ & $22( \pm 18.1)$ & 0.85 \\
\hline Infección nosocomial (\%) & $20(24)$ & $13(19)$ & $33(22 \%)$ & 0.36 \\
\hline
\end{tabular}


total pueden verse también en las Figuras 1 y 2 . La infección nosocomial fue frecuente en este grupo poblacional $21 \%$, con una tendencia mayor para los hombres (24\%), respecto de las mujeres (19\%) sin que la diferencia fuera estadísticamente significativa $(p=0.36)$. Entre las infecciones nosocomiales la neumonía fue las más frecuente $(45 \%)$ seguida de la infección asociada a dispositivo vascular (30\%) y menos frecuente la infección urinaria con $18 \%$ de los casos. Los hombres mostraron tener más casos de neumonía con 55\% del total de infecciones, respecto de $30 \%$ en mujeres $(p=$ $0.15)$ y las mujeres mostraron tener más casos de infección

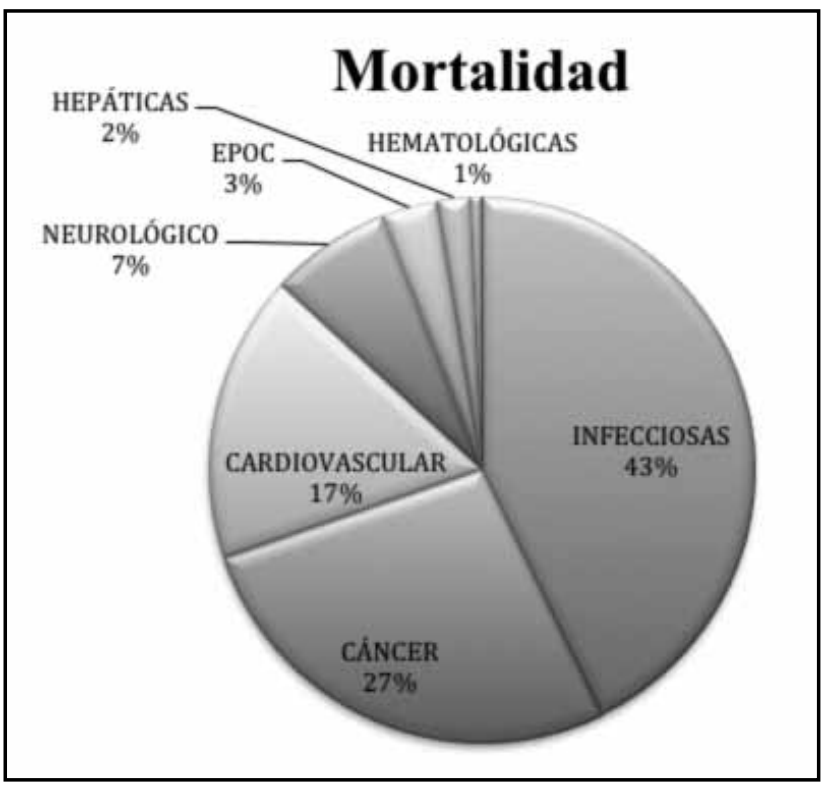

Figura 1. Causas de mortalidad medicina interna HUCSR. Año 2015. EPOC: enfermedad pulmonar obstructiva crónica. (Autor: Propiedad del Estudio). urinaria con $23 \%$ respecto de $15 \%$ en hombres $(p=0.56)$. En los casos de mortalidad relacionada con infección $(\mathrm{n}=$ 65), $65 \%$ tenían un Charlson de 2 o más, $33 \%$ tenían diabetes previa, $41 \%$ tenían neoplasia al momento del ingreso y todos los casos de VIH murieron de causa infecciosa. Entre los pacientes con infección nosocomial la estancia hospitalaria fue mucho mayor: 32 días; comparada con los pacientes que no tuvieron dicha patología cuya estancia media fue de 19 días $(p=0.002)$, así mismo entre las comorbilidades estos pacientes mostraron tener con mayor frecuencia ERC estadio 5 en $42 \%$ versus $19 \%(p=0.04)$ y $100 \%$ tenía hipoalbuminemia (albúmina $<3.5 \mathrm{~g} / \mathrm{dL}$ ) (Figura 3 ).

\section{Discusión}

La tasa de mortalidad hospitalaria de los pacientes de medicina interna del HUCSR fue de $8.3 \%$ para el año 2015. En general mucho más baja que la reportada para el promedio de los pacientes adultos de la ciudad de Bogotá, que en su último reporte del año 2015 se sitúa en 17\% para todos aquellos individuos que estuvieron más de 48 horas hospitalizados $(16,17)$. Esta diferencia, aunque importante no es del todo comparable, puesto que en principio nuestra evaluación incluye a la totalidad de pacientes entre los cuales una gran proporción tuvieron estancias hospitalarias menores de 48 horas, además que no incluyó a población general de pacientes quirúrgicos y tampoco a aquellos que ingresaron inicialmente por la UCI. Al revisar la literatura internacional, sin embargo, nuestra mortalidad está mucho más ajustada a la realidad de la región, toda vez que en las series revisadas, la mortalidad osciló entre $6-21 \%$ con un promedio de $13.1 \%(1-3,7,10,18-20)$, lo que característicamente es muy importante puesto que nos sitúa en el margen más bajo reportado. Sin embargo, estos resultados

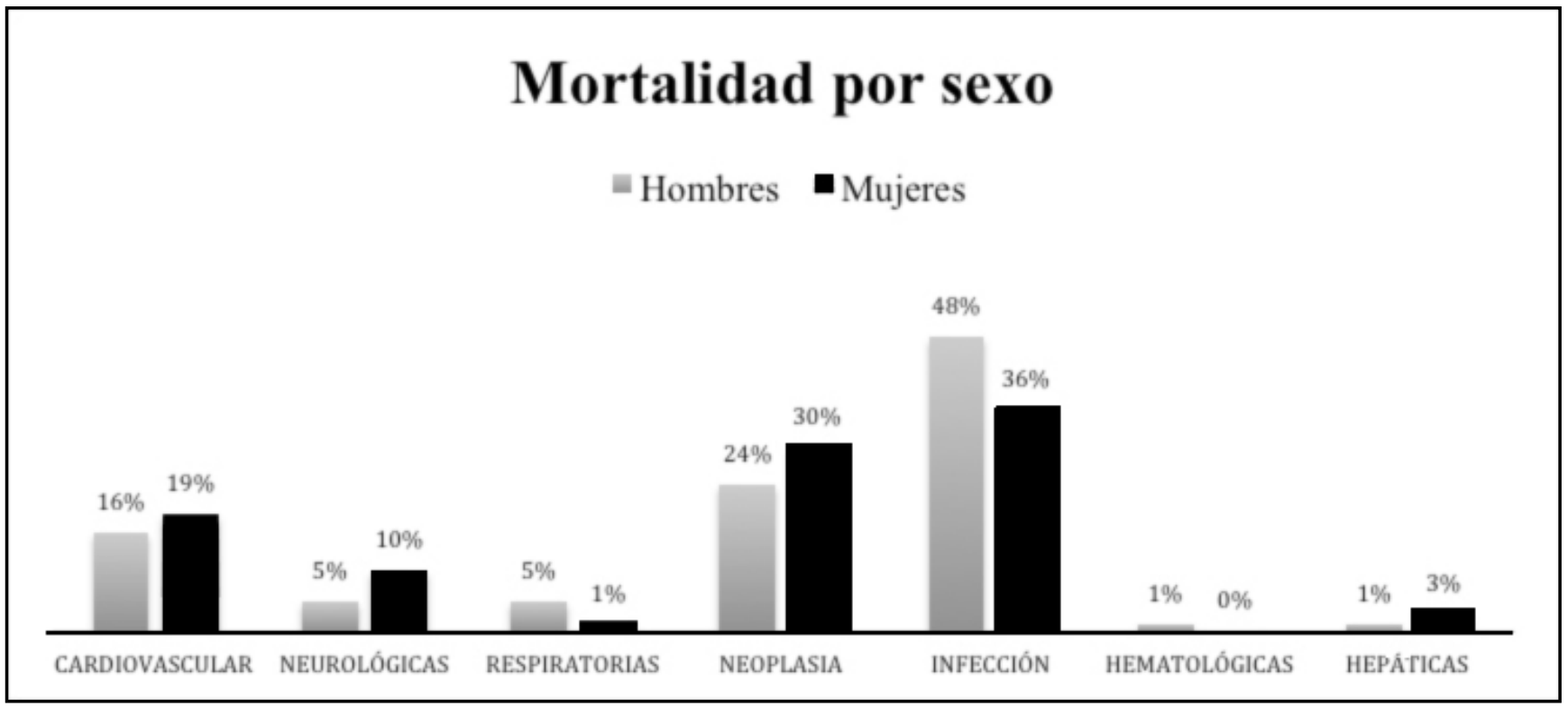

Figura 2. Causas de mortalidad por género. Medicina interna. HUCSR. Año 2015. (Autor: Propiedad del Estudio). 


\title{
Tipo de infección nosocomial
}

\author{
=Hombres $=$ Mujeres
}

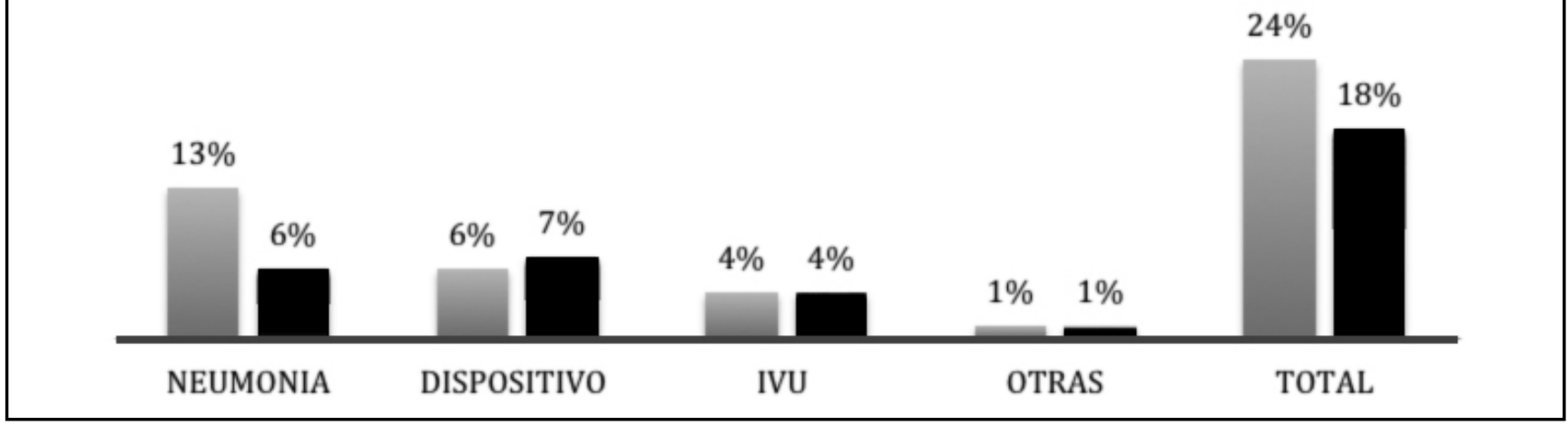

Figura 3. Infección nosocomial por género. Medicina interna. HUCSR. Año 2015. Dispositivo: infección por dispositivo intravascular. IVU: infección de vías urinarias. (Autor: Propiedad del Estudio).

no son suficientes del todo puesto que en la evaluación de la calidad de la atención son más importantes indicadores multidimensionales $(1,7,8)$ y no sólo el éxito en la sobrevida de los pacientes.

Nuestro estudio muestra la etiología infecciosa como la principal causa de muerte con $43 \%$, lo que contrasta con lo reportado en población general y en la mayoría de estudios revisados donde la principal causa fue la de origen cardiovascular $(2,3,6,8,12,18,20)$. De hecho, en su último informe sobre la calidad de la atención en salud, el ministerio de sanidad en Colombia reporta que la enfermedad cardiovascular es causal de $29 \%$ de todas las muertes de nuestra población adulta, seguida por el grupo de enfermedades crónicas como la EPOC y en tercer lugar al cáncer con un $17.5 \%$ (17). Estas diferencias tan importantes se explican por múltiples factores, en principio el HUCSR no es un centro de referencia de atención en patología cardiovascular, además está ubicado en la zona sur de la ciudad donde la población de influencia corresponde en su mayoría a estratos bajos con deficiencias importantes sanitarias y de acceso. Finalmente, es importante mencionar que el HUCSR en cambio es un centro de referencia de oncología y de hematología donde se atiende a población muy mórbida donde elementos tan frecuentes como el cáncer en $40 \%$ de los hospitalizados o la presencia de enfermedades como la diabetes mellitus en $28 \%$ pueden estar explicando la alta frecuencia de infecciones como causa final de muerte $(8,10,12,18,20)$. El único estudio en el que documentamos como primera causa de muerte la infección fue el de Ogun SA et al, el cual incluyó una población hospitalaria muy heterogénea y pediátrica lo que no lo hace del todo comparable a nuestra investigación, sin embargo, corresponde también a población de un país subdesarrollado (21).

Entre las comorbilidades encontradas, nuestra población se caracteriza por presentar una alta presencia de factores de riesgo cardiovascular como la hipertensión arterial (67\%), la diabetes mellitus (28\%) y la falla cardiaca (31\%). Esto es similar a lo encontrado en otras series $(1-3,7,10,16-18)$, sin embargo, nuestra segunda comorbilidad más frecuente fue el cáncer en $39 \%$ de la población, lo que se explica por la complejidad del hospital y el hecho de que sea un centro de atención de oncología de referencia en el sur de la ciudad. Esto nos hace un poco diferentes, puesto que las series revisadas muestran al cáncer como la tercera y cuarta comorbilidad predominante en medicina interna $(3,8,10,18)$. Así mismo al observar las causas de mortalidad en nuestra población es muy llamativo que la infección nosocomial siga siendo la primera causa de mortalidad hospitalaria $(21 \%)$, panorama que en países desarrollados disminuyó hace más de 20 años $(8,20)$. Sin embargo, en nuestro hospital creemos que está asociado a la alta frecuencia de cáncer, la carga de comorbilidades previa, la inmunosupresión por diabetes, la enfermedad renal y al hecho de que un alto porcentaje de la población que llega a nuestra institución es de bajos recursos, malnutrida y con dificultades para acceder al sistema de salud.

Se presentaron muy pocos casos de mortalidad en pacientes VIH positivos la mayoría de los cuales venía sin terapia HAART (Highly Active Antiretroviral Therapy). Al igual que en los otros estudios, no se encontró que una proporción importante de las causas de mortalidad fuera el VIH, excepto en el estudio africano de Ogun SA et al., donde nuevamente las condiciones locales así lo explican. De forma importante nuestro estudio muestra que la mortalidad de los pacientes con VIH fue en $100 \%$ de los casos de origen infeccioso, lo que además también contrasta con los resultados de la literatura donde en presencia de una buena terapia HAART la mayoría de estos pacientes mue- 
ren tardíamente de enfermedad cardiovascular (22). De los casos analizados de mortalidad por infección estos pacientes en su totalidad tuvieron hipoalbuminemia y entre los que desarrollaron infección nosocomial la ERC avanzada fue mucho más frecuente. Estos resultados son acordes a los encontrados en otros estudios, especialmente en pacientes de más de 80 años $(12,23,24)$.

Nuestro estudio tiene el valor de aportar información sobre la mortalidad del servicio de medicina interna de un hospital general de alta complejidad de la ciudad de Bogotá de forma reciente. Es punto de partida para conocer las características de la población que fallece, además para orientar estrategias hacia la identificación de grupos de riesgo con el ánimo de intervenir factores del huésped o de la atención modificables. Tiene como limitaciones que la recolección de la información se hizo de forma retrospectiva, donde con frecuencia se cometen sesgos de selección e información ligados a la calidad de los registros, además tampoco nuestro estudio permitió la evaluación de medidas de asociación para la identificación de factores de riesgo en la población. Para el futuro, creemos que este tipo de estudios son claves en el conocimiento de nuestra epidemiología local y esperamos que esta información sea útil a otros investigadores, creemos además que es importante que los hospitales de la ciudad hagan esfuerzos por desarrollar bases de datos locales que en conjunto con el sistema de sanidad del país fortalezcan nuestras propias redes de información.

\section{Conclusiones}

Nuestra investigación muestra como la mortalidad de los pacientes de medicina interna de un hospital de cuarto nivel del sur de la ciudad de Bogotá se sitúa alrededor de $8 \%$ y se acompaña frecuentemente de comorbilidades severas como el cáncer (39\%) y la falla cardiaca (30\%). En estos pacientes, la infección se encuentra entre las principales causas de muerte, lo que probablemente se explica por el perfil de comorbilidades y el bajo nivel socioeconómico en una población predominantemente del sur de la ciudad de Bogotá.

\section{Agradecimientos}

Los autores queremos agradecer a nuestros familiares, al Departamento de Medicina Interna del Hospital Universitario Clínica San Rafael de Bogotá, a la Fundación Universitaria Juan N. Corpas por su acompañamiento durante todo el proceso de elaboración del presente estudio.

\section{Referencias}

1. Sanclemente C, Barcons M, Moleiro MA, Alonso F, Pañella D, Carrera R, et al. [Hospital mortality in an Internal Medicine service]. An Med Interna [Internet]. 2004 Jul [cited 2015 May 23];21(7):317-21. Available from: http://www.ncbi.nlm. nih.gov/pubmed/15347235

2. Cinza Sanjurjo S, Cabarcos Ortiz de Barrón A, Nieto Pol E, Torre Carballada JA. [Analises of the mortality in aged in an Internal Medicine Department]. An Med Interna [Internet]. 2007 Feb [cited 2016 Apr 23];24(2):67-71. Available from: http:// www.ncbi.nlm.nih.gov/pubmed/17590091

3. Rayego Rodríguez J, Rodríguez-Vidigal FF, Mayoral Martín L, Alvarez-Oliva A, Najarro Díez F. [Hospital mortality in the Internal Medicine Services of a first level center]. An Med Interna [Internet]. 2006 Sep [cited 2016 Apr 23];23(9):406-10. Available from: http://www.ncbi.nlm.nih.gov/pubmed/17096601

4. Organización Mundial de la Salud. OMS I Mortalidad. [cited 2016 Apr 23]; Available from: http://www.who.int/topics/mortality/es/
5. Organización Mundial de la Salud. OMS $\mid$ Las 10 causas principales de defunción en el mundo. [cited 2016 Apr 23]; Available from: http://www.who.int/mediacentre/ factsheets/fs310/es/

6. Cinza Sanjurjo S, Cabarcos Ortiz de Barrón A, Nieto Pol E, Lorenzo Zúñiga V. [Epidemiologic analyses of elderly patients in an Internal Medicine department]. An Med Interna [Internet]. 2006 Sep [cited 2016 Apr 23];23(9):411-5. Available from: http://www.ncbi.nlm.nih.gov/pubmed/17096602

7. Barba R, Martínez JM, Zapatero A, Plaza S, Losa JE, Canora J, et al. Mortality and complications in very old patients $(90+)$ admitted to departments of internal medicine in Spain. Eur J Intern Med [Internet]. 2011 Feb [cited 2016 Apr 28];22(1):49-52. Available from: http://www.ncbi.nlm.nih.gov/pubmed/21238893

8. Viana A, Carballo F, Beato I, Domínguez E, Martínez C, de la Morena J. [An analysis of 14,000 patients admitted to an internal medicine service during 1982 1988]. An Med Interna [Internet]. 1990 Sep [cited 2016 Apr 29];7(9):459-62. Available from: http://www.ncbi.nlm.nih.gov/pubmed/2103286

9. Viana A, Delgado V, de la Morena J. [Mortality in the under-65 years of age in a teaching internal medicine service]. An Med Interna [Internet]. 1991 Jul [cited 2016 Apr 23];8(7):336-7. Available from: http://www.ncbi.nlm.nih.gov/pubmed/1932491

10. Matorras Galán P, Alonso López F, Daroca Pérez R, Lamelas Olarán JA, Díaz-Caneja Rodríguez N, Gancedo González Z. [Mortality at an internal medicine department of a tertiary hospital]. Rev clínica española [Internet]. $1989 \mathrm{Sep}$ [cited 2016 Apr 23];185(4):175-8. Available from: http://www.ncbi.nlm.nih.gov/ pubmed/2608963

11. Vega J, Parodi MJ, Puebla C. [Relationship between the cause of admission and the cause of death in patients admitted to an internal medicine departament]. Rev médica Chile [Internet]. 2004 Jun [cited 2016 Apr 28];132(6):707-17. Available from: http://www.ncbi.nlm.nih.gov/pubmed/15332372

12. Sonnenblick M, Raveh D, Gratch L, Yinnon A. Clinical and demographic characteristics of elderly patients hospitalised in an internal medicine department in Israel. Int J Clin Pract [Internet]. 2007 Feb [cited 2016 Apr 28];61(2):247-54. Available from: http://www.ncbi.nlm.nih.gov/pubmed/17263711

13. Francia E, Torres O, Laiz A, Ruiz D, Gich I, Casademont J. Ability of physiological parameters versus clinical categories to predict mortality on admission to an internal medicine ward. Eur J Intern Med [Internet]. 2009 Oct [cited $2016 \mathrm{Apr}$ 28];20(6):636-9. Available from: http://www.ncbi.nlm.nih.gov/pubmed/19782928

14. Leon Gordis. Epidemiology. Elsevier; 2014. 61-87 p.

15. Moreno Altamirano L. Epidemiología clínica. 3 ed. Moreno Altamirano L, editor. 2014.

16. Ministerio de Salud y Protección Social. Mortalidad General ONS [Internet] 2014 [cited 2016 Apr 23]. Available from: http://www.ins.gov.co/lineas-de-accion/ ons/mortalidad-general-en-colombia/dia3.html

17. Ministerio de Salud y Protección Social. Informe Nacional de Calidad de la Atención en Salud 2015 [Internet]. 2015 [cited 2018 Jun 10]. Available from: https:// www.minsalud.gov.co/sites/rid/Lists/BibliotecaDigital/RIDE/DE/DIJ/informe-nalcalidad-atencion-salud-2015.pdf

18. Del Río Fernández MC, Fernández Renedo A, de Frutos Arribas JF, Acebes Rey JM, Martín Escudero JC, Martínez Barrero F. [Mortality in an internal medicine department within a third-level hospital; twenty years experience]. An Med Interna [Internet]. 1998 Apr [cited 2016 Apr 23];15(4):197-201. Available from: http://www.ncbi.nlm.nih.gov/pubmed/9608063

19. Sicras Mainar A, Navarro Artieda R. [Measurement of mortality as the effect of hospital intervention at an internal medicine service]. An Med Interna [Internet]. 1992 Jan [cited 2016 Apr 23];9(1):21-9. Available from: http://www.ncbi.nlm.nih. gov/pubmed/1558910

20. De Escalante Yangüela B, Oncins Torres R, Lacasa Marzo J, Candel Calderón M,Sampedro Feliu JA. [Hospital mortality at the internal medicine service of a local hospital]. An Med Interna [Internet]. 1994 Aug [cited 2016 Apr 23];11(8):381-4. Available from: http://www.ncbi.nlm.nih.gov/pubmed/7772684

21. Ogun SA, Adelowo OO, Familoni OB, Jaiyesimi AE, Fakoya EA. Pattern and outcome of medical admissions at the Ogun State University Teaching Hospital, Sagamu--a three year review. West Afr J Med [Internet]. Jan [cited 2016 Apr 281;19(4):304-8. Available from: http://www.ncbi.nlm.nih.gov/pubmed/11391846

22. del Río Fernández MC, Fernández Renedo A, de Frutos Arribas JF, Sánchez González C, Martín Escudero JC, Martínez Barrero F. [Impact of AIDS on admissions and mortality in an Internal Medicine Department (1990-1995)]. Rev clínica española [Internet]. 1997 Apr [cited 2016 Apr 28];197(4):225-31. Available from: http://www.ncbi.nlm.nih.gov/pubmed/9254396

23. Perysinaki GS, Theodorakopoulou V, Bertsias A, Peteinaraki E, TsachakisMueck N, Giaka M, et al. Almost half of octogenarians and nonagenarians admitted acutely to internal medicine ward die during admission or within 6 months after discharge: time to redefine treatment goals? J Am Geriatr Soc [Internet]. 2015 Feb [cited 2016 Apr 28];63(2):380-3. Available from: http://www.ncbi.nlm.nih.gov/ pubmed/25688610

24. Zafrir B, Laor A, Bitterman H. Nonagenarians in internal medicine: characteristics, outcomes and predictors for in-hospital and post-discharge mortality. Isr Med Assoc $J$ [Internet]. 2010 Jan [cited 2016 Apr 28];12(1):10-5. Available from: http://www. ncbi.nlm.nih.gov/pubmed/20450122 